\title{
Quantum Phase Transition in the Skyrmion Lattice
}

\author{
Yu. V. Nazarov and A. V. Khaetskii* \\ Faculty of Applied Physics and DIMES, Delft University of Technology, Lorentzweg 1, 2628 CJ Delft, The Netherlands
} (Received 13 March 1997)

\begin{abstract}
We investigate the ground state of the $2 \mathrm{D}$ electron gas in the quantum Hall regime at a filling factory slightly deviating from unity, which can be viewed as a sparse lattice of Skyrmions. We have found that in the low density limit Skyrmions are bound in pairs, the latter forming an actual lattice. We have shown that with an increase in density the lattice undergoes a quantum phase transition, which is analogous to the superconducting phase transition in Josephson junction arrays. [S0031-9007(97)05060-6]
\end{abstract}

PACS numbers: 73.40.Hm, 12.39.Dc, 73.20.Dx

The Skyrmion [1] has been a favorite model particle of field theorists for decades. Only recently has it been understood that the low-lying charged excitations of the integer quantum Hall ground state may be Skyrmions ([2], see [3] for review). This interesting development prompted intensive experimental studies $[4,5]$ which proved these exotic particles to be real. A Skyrmion can be viewed as a topologically nontrivial distortion of the spins of many ground state electrons which makes its spin much bigger than unity, although the Skyrmion bears a unitary charge. The size of the Skyrmions is controlled by a parameter $\tilde{g}$, which is the ratio of the Zeeman energy to exchange energy per particle. The smaller the $\tilde{g}$, the bigger the Skyrmion.

If the filling factor deviates slightly from unity, the lowlying charged excitations must appear at the background of the quantum Hall state to compensate for the excess charge. Therefore, the deviation of the filling factor sets the Skyrmion concentration and the many-Skyrmion ground state can easily be made by changing either the magnetic field or the electron density of the 2D gas. Two studies of the many-Skyrmion ground state at finite $\tilde{g}$ have been recently reported [6,7]. Despite the different methods applied and the different results obtained, it was assumed in both studies that Skyrmions form a plain regular lattice. In both studies no quantum effects were taken into account.

Our results demonstrate that the formation and development of the multi-Skyrmion state is more complicated and interesting than it has been previously assumed. We show that at low concentrations Skyrmions always appear in pairs to form a triangular Wigner lattice. With increasing concentration, the distance between pairs decreases and the interaction between the pairs strengthens. This leads to a quantum transition in the Skyrmion lattice. Below the transition point, the $z$ component of the spin of each pair is well defined. We call the variable that is canonically conjugate to the $z$ component of the spin the phase. Above the transition point, it is this phase that is well defined.

We begin with the energy functional that describes spin textures in the limit of small $\tilde{g}$ (see, e.g., Refs. [2,7]).

$$
\begin{aligned}
E= & E_{\text {stiff }}+E_{Z}+E_{\text {Coulomb }} \\
= & \int d^{2} r\left[\frac{\rho_{s}}{2}\left|\partial_{\mu} \mathbf{m}\right|^{2}+\frac{|g| \mu_{B} B n_{L}}{2}\left(1+m_{z}\right)\right] \\
& +\frac{1}{2} \int d^{2} r \int d^{2} r^{\prime} q(\mathbf{r}) \frac{e^{2}}{\epsilon\left|\mathbf{r}-\mathbf{r}^{\prime}\right|} q\left(\mathbf{r}^{\prime}\right) .
\end{aligned}
$$

It contains three terms describing spin stiffness, Zeeman energy, and electrostatic energy, respectively, and depends on $\mathbf{m}(\mathbf{r})$, a unitary vector characterizing local spin density. We adopt the microscopic expression [8] for the spin stiffness at filling factor 1: $\rho_{s}=$ $(1 / 16 \sqrt{2 \pi})\left(e^{2} / \epsilon \lambda\right), \lambda$ being the magnetic length. In the Zeeman term, $g$ stands for the electron $g$ factor, and $\mu_{B}$ is the Bohr magneton. In the electrostatic term, $q(\mathbf{r})$ presents the deviation of the electron density from the uniform background density $n_{L}=1 / 2 \pi \lambda^{2}, q$ being related to the density of the topological charge [2]

$$
q=-\frac{1}{8 \pi} \epsilon_{\mu \nu}\left(\mathbf{m}\left[\partial_{\mu} \mathbf{m} \times \partial_{\nu} \mathbf{m}\right]\right) .
$$

The precise definition of $\tilde{g}$ reads $\tilde{g} \equiv g \mu_{B} B / E_{\text {ex }}, E_{\text {ex }} \equiv$ $e^{2} / \epsilon \lambda$.

The stiffness term dominates the energy and must be the first to be minimized. It has been shown by Belavin and Polyakov [9] that the textures minimizing the stiffness term are highly degenerate. They parametrized the spin density as follows:

$$
\begin{aligned}
W & =\frac{m_{x}+i m_{y}}{1-m_{z}}, \quad m_{x}+i m_{y}=\frac{2 W}{1+|W|^{2}}, \\
m_{z} & =\frac{|W|^{2}-1}{|W|^{2}+1} .
\end{aligned}
$$

It turns out that all the textures corresponding to any analytical function $W(z \equiv x+i y)$ with $N$ poles possess the same energy $N \sqrt{\pi / 32} E_{\text {ex }}$. Neither the poles (Skyrmion positions) nor the pole residuals (Skyrmion radii) are fixed. These are determined by the interplay of the weaker interactions, namely, Zeeman and electrostatic energy.

Let us start with a single Skyrmion [2]. We take $W(z)=a / z, a$ being the Skyrmion radius to be evaluated. 
The Coulomb energy is given by $E_{Q}=\left(3 \pi^{2} / 64\right) e^{2} / \epsilon a$. The integral that gives the Zeeman energy appears to diverge logarithmically at long distances from the core, since the $x, y$ spin components decrease very slowly with increasing distance. A slightly improved calculation shows that the divergence is to be cut at $r_{s} \simeq \lambda \tilde{g}^{-1 / 2}$, and the Zeeman energy reads $E_{Z}=g \mu_{B} B\left(a^{2} / \lambda^{2}\right) \ln \left(r_{s} / a\right)$ provided $a \ll r_{s}$. The minimization of the total energy with respect to $a$ yields $a \simeq \lambda[\ln (1 / \tilde{g}) \tilde{g}]^{-1 / 3}$, with $a \ll r_{s}$ as required.

Let us consider now two Skyrmions with opposite phases,

$$
W(z)=\frac{a}{z+R}-\frac{a}{z-R},
$$

with the minus sign before the second term accounting for the phase shift. We assume that $R \ll r_{s}$. We see that the $x, y$ spin components are effectively quenched at $|z| \gg$ $R$, which lowers the Zeeman energy. For well-separated Skyrmions $(a \ll R)$ the logarithmical divergency is cut off at distances of the order of $R$, and the Zeeman energy reads $2 g \mu_{B} B\left(a^{2} / \lambda^{2}\right) \ln (R / a)$. This results in an attractive force between Skyrmions $\propto 1 / R$. At long distances it always prevails over Coulomb repulsion, so that the Skyrmions are bound to form pairs. To find exactly how they are bound, we have studied the question numerically. At a given Skyrmion separation $R$, we minimize two-Skyrmion energy with respect to $a$. Our results are plotted in Fig. 1. It appears that the minimum energy is achieved at zero separation, corresponding to $W(z)=b^{2} / z^{2}$. Two Skyrmions merge as is sketched in Fig. 1. The optimal value of $|b|$ is $b_{0} \simeq \lambda / \tilde{g}^{1 / 3}$. The Skyrmion pair bears a big spin, $S_{0} \simeq 0.78 / \tilde{g}^{2 / 3}$. We checked numerically that the Skyrmion attraction saturates, so that there is no further binding of pairs.

While at $R<r_{s}$ the interaction between Skyrmions is attractive, for $R>r_{s}$ the Zeeman part of interaction dies out and Coulomb repulsion prevails. This suggests that the curve plotted in Fig. 1 has a maximum at $R \simeq r_{s}$ and

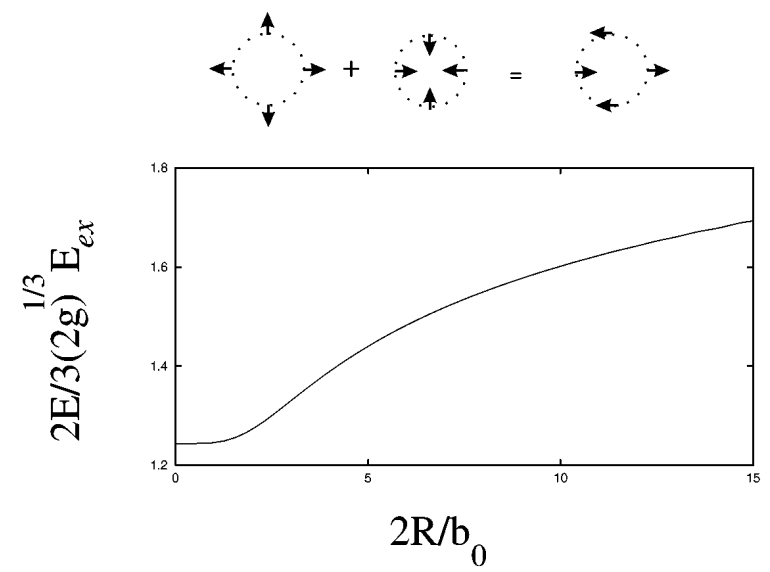

FIG. 1. Binding of two Skyrmions. The two-skyrmion energy as a function of their separation has a minimum at zero separation. therefore there is a barrier to the binding of two Skyrmions. The binding is energetically favorable provided $E(R=$ $0)<E(R=\infty)$. Within the applicability of the continuous approach we use, that is, $\tilde{g} \rightarrow 0$, this is always true. The situation might change at some critical value of $\tilde{g}$ to be determined from microscopic theory.

Therefore, we have shown that charged excitations with lowest energy are eventually Skyrmion pairs bearing charge $\pm 2 e$. We proceed now with analyzing the manySkyrmion ground state [10]. Let us compare the Zeeman and Coulomb contributions to the interaction between pairs. The latter prevails provided the separation between pairs exceeds their equilibrium radius $b_{0}$. Hence, the pairs behave very much like pointlike charges and form a triangular Wigner lattice to minimize their Coulomb energy. Such an arrangement persists up to relatively high Skyrmion densities $n_{\text {sky }} / n_{\mathrm{el}} \simeq\left(b_{0} / \lambda\right)^{-2} \simeq \tilde{g}^{2 / 3}$ where they begin to overlap. Most probably, the overlap will depair Skyrmions, and one of the single-Skyrmion lattices proposed could be realized at higher densities. In the present paper, we restrict our attention to lower densities, where the pairs certainly form a triangular lattice.

To reveal the interesting physics that persists even in this limiting case, let us note that the two-Skyrmion solution found is infinitely degenerate. All the spins can be rotated about the $z$ axis by an arbitrary angle yielding texture of the same energy. Or, alternatively, we can multiply $W(z)$ by an arbitrary phase factor $\exp (i \phi)$. We will call the variable parametrizing all of the degenerate textures the phase. This degeneracy is not physical and arises from the fact that we regard the pair, which is a quantum particle, as a classical object.

Let us consider quantization of an isolated pair. We note that the shift in the phase space, $\phi \rightarrow \phi+\theta$, is just a rotation in the spin space about the $z$ axis represented by the quantum mechanical operator $\exp \left(-i \theta \hat{S}_{z}\right)$. Here $\hat{S}_{z}$ stands for the total spin of the Skyrmion. Expanding near $\theta=0$, we find that $S_{z}=-i \partial / \partial \phi$. That leads us to the following important conclusion:

$$
\left[\hat{\phi}, \hat{S}_{z}\right]=i,
$$

the phase and the total spin of the pair are conjugate variables satisfying the Heisenberg uncertainty relation. Owing to the degeneracy with respect to the phase, physical isolated Skyrmion pairs have no definite phase but rather possess a definite integer $z$ component of the spin. This picture has very much in common with the Coulomb blockade in a superconducting island $[11,12]$ that has no definite superconducting phase but rather a certain integer charge.

Since the total spin of the pair is a function of its radius $b$, as well as its energy, the spin quantization leads to the energy quantization of pair states. The spectrum can be obtained from classical dependences $E(b)$ and $S_{z}(b)$. Near the minimum energy, it reads

$$
E=E_{0}+E_{c}\left(S_{z}-S_{0}\right)^{2}, \quad E_{c} \equiv \frac{3}{4} \frac{\tilde{g} E_{\mathrm{ex}}}{S_{0}} .
$$


Here $E_{0}, S_{0}$ are the optimal classical energy and spin, respectively. Let us note that $S_{0}$ is a continuous function of $\tilde{g}$, to be contrasted with integer $S_{z}$. If we change $\tilde{g}$, $S_{0}$ changes continuously, whereas the optimal $S_{z}$ jumps between the integer values. At the point of jump, the energies of the two states with different $S_{z}$ match, providing extra degeneracy.

This is reminiscent of the Coulomb blockade phenomenon in Josephson arrays [12]. Josephson arrays consist of superconducting islands; the number of discrete charges in each island is canonically conjugate to its superconducting phase. The actual state of the array is determined by the interplay of the charging energy and the Josephson coupling. If charging energy dominates, the array is in the insulating phase. There is a gap for charged excitations: One has to pay charging energy in order to add a discrete charge to or extract it from the array. If the Josephson coupling increases, the quantum phase transition to the superconducting phase takes place. A long-range phase order appears and the gap vanishes.

We expect similar behavior in Skyrmion lattices, with discrete charge replaced by discrete $S_{z}$. At very low Skyrmion concentrations the pairs can be regarded as isolated ones, each having a well-defined spin and a strongly fluctuating phase. The $x, y$ components of the local spin density are undefined and have zero average, as is sketched in Fig. 2(a). There is a "spin gap" in this "insulating" phase: One has to pay energy of the order of $E_{c}$ to increase or decrease the spin of the system by unity. If we increase the Skyrmion concentration, the pairs come closer to each other. This increases coupling, tending to fix the phases of the neighboring pairs. Therefore, we expect a quantum transition to the "superconducting" phase. At the transition point the spin gap vanishes. Instead, a long-range phase order appears, i.e., the $x, y$ components of the local spin density become nonzero.

To give a quantitative description, we evaluate first the phase-dependent interaction between pairs. It appears that this interaction measures up $E_{c}$ at inter-Skyrmion dis-

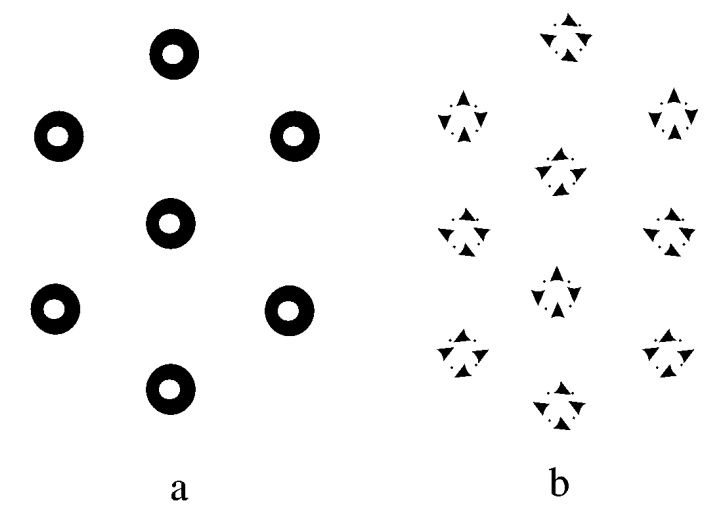

FIG. 2. Two phases of the Skyrmion lattice. (a) In the insulating phase, the Skyrmion phase fluctuates strongly so that the $x, y$ spin components have zero average (b) in the superconducting phase, the Skyrmion phases are fixed and antiferromagnetically arranged. tances much larger than the screening radius $r_{s},\left(r_{s} / \lambda\right)^{2}=$ $\sqrt{\pi} / 4 \sqrt{2} \tilde{g}$. We therefore evaluate it for interpair distances $r$ in the region $r \gg r_{s}, b$. We expand the energy functional up to terms quadratic in $s_{x, y}$ and minimize the resulting expression matching $s_{x, y}$ near the pair cores with the asymptotics of $W(z)$. This yields

$$
\begin{aligned}
E_{\mathrm{int}} & =E_{J} \cos \left(\phi_{1}-\phi_{2}\right), \\
E_{J} & =g \mu_{B} B \frac{b_{0}^{4}}{r_{s}^{4}} \exp \left(-\frac{r}{r_{s}}\right)\left(\frac{r_{s}}{\lambda^{2}} \sqrt{\pi r_{s} r / 2}\right) .
\end{aligned}
$$

As a result, we have the following many-body Hamiltonian, which takes into account "charging" and Josephson energies:

$$
\begin{aligned}
\hat{H}= & \sum_{i} E_{c}\left(-i \frac{\partial}{\partial \phi_{i}}-S_{0}\right)^{2} \\
& +\frac{1}{2} \sum_{i j}^{\prime} E_{J} \cos \left(\phi_{i}-\phi_{j}\right),
\end{aligned}
$$

where $E_{c}$ is given by Eq. (6) and $E_{J}>0$ is given by Eq. (7). The indices label the sites of the triangular lattice, the prime restricting the sum over $j$ to the nearest neighbors of a certain site. Note that in our case the "Josephson" coupling has an antiferromagnetic sign, to be contrasted with Josephson arrays. In the limit $E_{J} \gg E_{c}$ the phases become well defined, and we can neglect the charging term. The Hamiltonian reduces to the energy functional. In the ground state, the lattice subdivides into three sublattices [13]. The phases of the sublattices are rotated by $2 \pi / 3$ with respect to each other [Fig. 2(b)].

$\mathrm{We}$ are interested in the transition characteristics. Since the exact solution of the Hamiltonian is beyond reach, the transition point is found in the Hartree approximation. We approximate the exact ground state wave function by the product of site wave functions,

$$
\Psi\left(\phi_{1}, \ldots, \phi_{N}\right)=\prod_{i=1}^{N} \Psi_{i}\left(\phi_{i}\right)
$$

with $\Psi_{i}$ to be determined from the minimum energy principle. The site wave functions then satisfy the following mean-field Hamiltonian:

$$
\begin{aligned}
\hat{H}_{\mathrm{MF}}= & \sum_{i} E_{c}\left(-i \frac{\partial}{\partial \phi_{i}}-S_{0}\right)^{2} \\
& +\frac{1}{4} E_{J} \sum_{i j}^{\prime}\left[2 n_{j} \exp \left(-i \phi_{i}\right)-n_{i} n_{j}^{*}+\text { c.c. }\right],
\end{aligned}
$$

where we introduce the order parameter

$$
n_{i}=\int_{0}^{2 \pi} \exp (i \phi)\left|\Psi_{i}(\phi)\right|^{2} d \phi
$$

at each site. We assume that near the transition point the order parameter has the same symmetry as for $E_{J} \gg$ $E_{c}$. The nontrivial solution for the order parameter first appears at

$$
E_{J}^{0}=\frac{E_{c}}{3}\left\{1-4\left(\left[S_{0}+1 / 2\right]-S_{0}\right)^{2}\right\},
$$


this equation determining the transition point. Making use of Eqs. (6), (7), and (10) we find that the transition occurs at Skyrmion density $n_{\text {sky }} \sim r_{s}^{-2} \ln ^{-2}(1 / \tilde{g})$. To give more detailed predictions, we plot the critical Skyrmion density versus $\tilde{g}$ in the realistic range of this parameter. We assume that in this range the continuous approach to Skyrmions still gives reasonable results. The curve exhibits sharp drops each time the energies of two spin states match, since this favors spin fluctuations and, consequently, facilitates phase ordering.

To observe the transition experimentally, one should measure either the spin gap or the local magnetization parallel to the plane. These quantities vanish and appear, respectively, above the transition point. Both quantities are likely to be obtained by magnetic resonance measurement. The experiment should be performed at temperatures lower than the typical energy scale involved, $E_{c}$. Our estimations for $n_{\mathrm{el}}=10^{16} \mathrm{~m}^{-2}$, $\tilde{g}=0.025$ gives $E_{c} / k_{B} \simeq 1 \mathrm{~K}$. Fine-tuning of the filling factor in the range of 0.01 is required. In our calculation we used the values of the microscopic parameters that have been evaluated under simplified assumptions. For instance, Landau level mixing and the finite width of the electronic wave function in the growth direction have not been taken into account. That might complicate the quantitative comparison with experiment, although we do not expect the qualitative conclusions to change. Our model does not explicitly include disorder. However, we expect that the quantum transition occurs even in disordered heterostructures, provided the Skyrmions survive. Also, the binding of two Skyrmions is not necessary for the quantum transition. The disorder can trap the Skyrmions in random potential minima, thus preventing the formation of any regular lattice. However, it cannot pin the Skyrmion phase; this can be done only by inter-Skyrmion interactions with increasing concentration. Hence, we expect the quantum transition at $n_{\mathrm{sky}} / n_{\mathrm{el}} \simeq \tilde{g}$

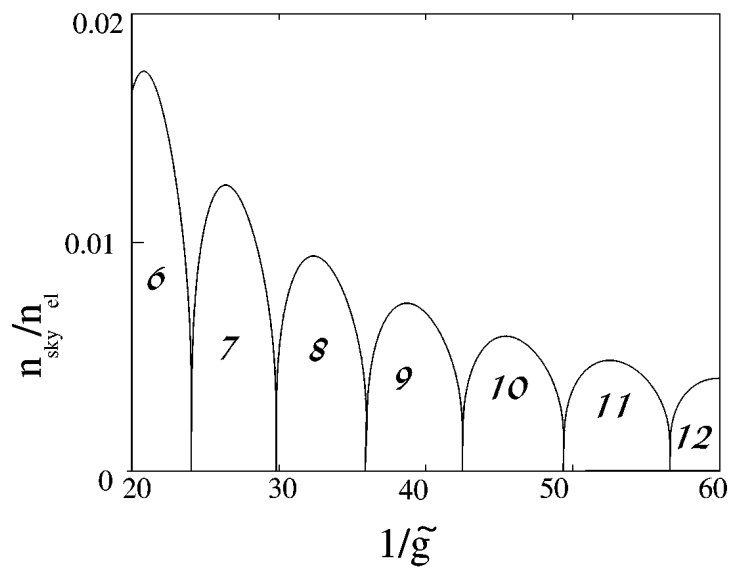

FIG. 3. The quantum phase transition. The critical Skyrmion density is plotted in the region of realistic $\tilde{g}$. Above the line, the system is in the superconducting state. The numbers denote the equilibrium spin of isolated Skyrmion pairs at given $\tilde{g}$. The critical line drops to zero each time the spin state changes. even for a disordered and/or unbound arrangement of Skyrmions.

In conclusion, we have investigated the manySkyrmion ground state at a low-Skyrmion concentration and have shown that Skyrmions are bound in pairs to form a triangular Wigner lattice. The quantum phase transition occurs in this lattice at a certain Skyrmion concentration.

The authors wish to thank G.E.W. Bauer, L.P. Kouwenhoven, M. V. Feigelman, and S.A. Kivelson for very constructive discussions of our results. We acknowledge the financial support of "Nederlandse Organisatie voor Wetenschappelijk Onderzoek (NWO)" that made this work possible. A. V.K. acknowledges the kind hospitality of Professor G.E.W. Bauer and Dr. Yu. V. Nazarov during his visit to Delft TU.

*Permanent address: Institute of Microelectronics Technology, Russian Academy of Sciences, 142432, Chernogolovka, Moscow District, Russia.

[1] T.H. R. Skyrme, Proc. R. Soc. London A 262, 233 (1961).

[2] S. L. Sondhi, A. Karlshede, S. A. Kivelson, and E.H. Rezayi, Phys. Rev. B 47, 16419 (1993).

[3] A.H. MacDonald, in Quantum Transport in Semiconductor Heterostructures, edited by B. Kramer (Kluwer, Dordrecht, 1996), p. 110.

[4] S. E. Barrett, G. Dabbagh, L. N. Pfeiffer, K. W. West, and R. Tysko, Phys. Rev. Lett. 74, 5112 (1995); E. H. Aifer, D. A. Broido, and B. B. Goldberg, Phys. Rev. Lett. 76, 680 (1996); A. Schmeller, J. P. Eisenstein, L. N. Pfeiffer, and K. W. West, Phys. Rev. Lett. 75, 4290 (1995).

[5] D. K. Maude, M. Potemski, J.C. Portal, M. Henini, L. Eaves, G. Hill, and M. A. Pate, Phys. Rev. Lett. 77, 4604 (1996).

[6] L. Brey, H. A. Fertig, R. Cote, and A.H. MacDonald, Phys. Rev. Lett. 75, 2562 (1995).

[7] A. G. Green, I. I. Kogan, and A. M. Tsvelik, Phys. Rev. B 54, 6838 (1996).

[8] Yu. A. Bychkov, S. V. Iordansky, and G. M. Eliashberg, JETP Lett. 33, 152 (1981); Yu. A. Bychkov, Y. Maniv, and I.D. Wagner, Phys. Rev. B 53, 10148 (1996); K. Moon, H. Mori, Kun Yang, S. M. Girvin, A.H. MacDonald, L. Zheng, D. Yoshioka, and Shou-Cheng Zhang, Phys. Rev. B 51, 5138 (1995).

[9] A. A. Belavin and A. M. Polyakov, JETP Lett. 22, 503 (1975).

[10] The Skyrmion pairs are bosons so that they can form a superconducting condensate. Whatever the fascinating opportunity, this does not happen since they are effectively localized by a strong magnetic field. Or, in other terms, the potential superconductivity is suppressed by the field.

[11] K. B. Efetov, Sov. Phys. JETP 51, 1015 (1980).

[12] J.E. Mooij and G. Schön, in Single Charge Tunneling, edited by H. Grabert and M. Devoret (Plenum, New York, 1992), p. 275.

[13] G. H. Wannier, Phys. Rev. 79, 357 (1950); D. H. Lee, J. D. Joannopoulous, J. W. Negele, and D. P. Landau, Phys. Rev. B 33, 450 (1986). 